\title{
No-go theorem on spontaneous parity breaking revisited
}

\author{
V. Azcoiti ${ }^{\mathrm{a}}$, A. Galante ${ }^{a *}$ \\ ${ }^{\text {a}}$ Departamento de Física Teórica, Facultad de Ciencias,Universidad de Zaragoza,50009 Zaragoza,Spain
}

An essential assumption in the Vafa and Witten's theorem on P and CT realization in vector-like theories concerns the existence of a free energy density in Euclidean space in the presence of any external hermitian symmetry breaking source. We show how this requires the previous assumption that the symmetry is realized in the vacuum. Even if Vafa and Witten's conjecture is plausible, actually a theorem is still lacking.

A few years ago Vafa and Witten gave an argument against spontaneous breaking of parity in vector-like parity-conserving theories as QCD [1]. The main point in their proof was the crucial observation that any arbitrary hermitian local order parameter $X$ constructed from Bose fields should be proportional to an odd power of the four indices antisymmetric tensor $\epsilon^{\mu \nu \rho \eta}$ and therefore would pick-up a factor of $i$ under Wick rotation. The addition of an external symmetry breaking field $\lambda X$ to the Lagrangian in Minkowski space becomes then a pure phase factor in the pathintegral definition of the partition function in Euclidean space. But a pure phase factor in the integrand of a partition function with positive definite integration measure can only increase the vacuum energy density and their conclusion was that, in such a situation, the mean value of the order parameter should vanish in the limit of vanishing symmetry breaking field.

A weak point in this simple and nice argument is the assumption that the vacuum energy density (equivalently, the free energy density) is well defined when the symmetry breaking external field $\lambda$ is not zero.

We want to show here how Vafa and Witten's argument breaks down if parity is spontaneously broken [2]. In other words, the assumption that the vacuum energy density is well defined at nonvanishing $\lambda$ requires the previous assumption that parity is not spontaneously broken.

To demonstrate it we will consider the probability distribution function (p.d.f.) of any or-

\footnotetext{
${ }^{*}$ Talk presented by A. Galante
}

der parameter for parity in vector-like parityconserving theories. The analysis of the p.d.f. of the order parameter in the symmetric model has been extensively and successful used to investigate spin systems [3], spin glasses [泊] and quantum field theories with fermionic degrees of freedom [5.

In our approach, in order to work with well defined mathematical objects, we will use the lattice regularization scheme and assume that the lattice regularized action preserves, as for KogutSusskind fermions, the positivity of the determinant of the Dirac operator. The other essential assumption we use is that the hermitian P-nonconserving order parameter is a local operator constructed from Bose fields and therefore, as any intensive operator, it does not fluctuate in a pure vacuum state. This property is equivalent to the statement that all connected correlation functions verify cluster property in a pure vacuum state.

The Euclidean path integral formula for the partition function is

$\mathcal{Z}=\int d A_{\mu}^{a} d \bar{\psi} d \psi e^{-\int d^{4} x(L(x)+i \lambda X(x))}$

where following Vafa and Witten we have exhibited the factor of $i$ that arises from Wick rotation, i.e. $\mathrm{X}$ in (1) is real.

Using the p.d.f. of the order parameter $X$, we can write the partition function as

$\mathcal{Z}(\lambda)=\mathcal{Z}(0) \int d \tilde{X} P(\tilde{X}, V) e^{-i \lambda V \tilde{X}}$

where $V$ in (2) is the number of lattice sites, 
$P(\tilde{X}, V)$ is the p.d.f. of $X$ at a given lattice volume

$$
\begin{aligned}
& P(\tilde{X}, V)= \\
& \frac{\int d A_{\mu}^{a} d \bar{\psi} d \psi e^{-\int d^{4} x L(x)} \delta\left(\bar{X}\left(A_{\mu}^{a}\right)-\tilde{X}\right)}{\int d A_{\mu}^{a} d \bar{\psi} d \bar{\psi} e^{-\int d^{4} x L(x)}}
\end{aligned}
$$

and

$$
\bar{X}\left(A_{\mu}^{a}\right)=\frac{1}{V} \int d^{4} x X(x)
$$

Notice that, since the integration measure in (3) is positive or at least semi-positive definite, $P(\tilde{X}, V)$ is a true well normalized p.d.f.

Let us assume that parity is spontaneously broken. In the simplest case in which there is no extra vacuum degeneracy due to spontaneous breakdown of some other symmetry, we will have two vacuum states as corresponds to a discrete $Z_{2}$ symmetry. Since $X$ is an intensive operator, the p.d.f. of $X$ will be, in the thermodynamical limit, the sum of two $\delta$ distributions:

$$
\lim _{V \rightarrow \infty} P(\tilde{X}, V)=\frac{1}{2} \delta(\tilde{X}-a)+\frac{1}{2} \delta(\tilde{X}+a)
$$

At any finite volume, $P(\tilde{X}, V)$ will be some symmetric function $(P(\tilde{X}, V)=P(-\tilde{X}, V))$ developing a two peak structure at $\tilde{X}= \pm a$ and approaching (4) in the infinite volume limit.

Due to the symmetry of $P(\tilde{X}, V)$ we can write the partition function as

$$
\mathcal{Z}(\lambda)=2 \mathcal{Z}(0) \operatorname{Re} \int_{0}^{\infty} P(\tilde{X}, V) e^{-i \lambda V \tilde{X}} d \tilde{X}
$$

and if we pick up a factor of $e^{-i \lambda V a}$ after simple algebra we get:

$$
\mathcal{Z}(\lambda) /(2 \mathcal{Z}(0))=
$$

$$
\begin{array}{r}
\cos (\lambda V a) \int_{0}^{\infty} P(\tilde{X}, V) \cos (\lambda V(\tilde{X}-a)) d \tilde{X} \\
-\sin (\lambda V a) \int_{0}^{\infty} P(\tilde{X}, V) \sin (\lambda V(\tilde{X}-a)) d \tilde{X}
\end{array}
$$

The relevant zeroes of the partition function in $\lambda$ can be obtained as the solutions of the following equation:

$$
\cot (\lambda V a)=\frac{\int_{0}^{\infty} P(\tilde{X}, V) \sin (\lambda V(\tilde{X}-a)) d \tilde{X}}{\int_{0}^{\infty} P(\tilde{X}, V) \cos (\lambda V(\tilde{X}-a)) d \tilde{X}}(6)
$$

Let us assume for a while that the denominator in (6) is constant at large $V$. Since the absolute value of the numerator is bounded by 1 , the partition function will have an infinite number of zeroes approaching the origin $(\lambda=0)$ with velocity $V$. In such a situation the free energy density does not converge in the infinite volume limit.

But this is essentially what happens in the actual case. In fact if we consider the integral in (6)

$f(\lambda V, V)=\int_{0}^{\infty} P(\tilde{X}, V) \cos (\lambda V(\bar{X}-a)) d \tilde{X}(7)$

as a function of $\lambda V$ and $V$ it is easy to check that the derivative of $f(\lambda V, V)$ respect to $\lambda V$ vanishes in the large volume limit due to the fact that $P(\tilde{X}, V)$ develops a $\delta(\tilde{X}-a)$ in the infinite volume limit. At fixed large volumes $V, f(\lambda V, V)$ as function of $\lambda V$ is an almost constant non-vanishing function (it takes the value of $1 / 2$ at $\lambda V=0$ ). The previous result on the zeroes of the partition function in $\lambda$ remains therefore unchanged.

To illustrate this result with an example, let us take for $P(\tilde{X}, V)$ a double gaussian distribution

$$
\begin{aligned}
& P(\tilde{X}, V)= \\
& \quad \frac{1}{2}\left(\frac{V}{\pi}\right)^{1 / 2}\left(e^{-V(\tilde{X}-a)^{2}}+e^{-V(\tilde{X}+a)^{2}}\right)
\end{aligned}
$$

which gives for the partition function

$$
\mathcal{Z}(\lambda)=Z(0) \cos (\lambda V a) e^{-\frac{1}{4} \lambda^{2} V}
$$

and for the mean value of the order parameter

$$
<i X>=\frac{1}{2} \lambda+\tan (\lambda a V) a
$$

The zeroes structure of the partition function is evident in (9) and consequently the mean value 
of the order parameter (10) is not defined in the thermodynamical limit. Notice also that if $a=0$ (symmetric vacuum), the free energy density is well defined at any $\lambda$ and then Vafa and Witten's argument applies.

In conclusion we have shown that an essential assumption in the Vafa and Witten's theorem on $\mathrm{P}$ and $\mathrm{CT}$ realization in vector-like theories, namely the existence of a free energy density in Euclidean space in the presence of any external hermitian symmetry breaking source, does not apply if the symmetry is spontaneously broken. The assumption that the free energy density is well defined requires the previous assumption that the symmetry is realized in the vacuum.

To clarify this point let us discuss a simple model which, as vector-like theories, has a positive definite integration measure and, after the introduction of an imaginary order parameter, a complex action: the Ising model in presence of an imaginary external magnetic field. This model verifies all the conditions of Vafa-Witten theorem. If we assume that the free energy density exists, we will conclude that the $Z_{2}$ symmetry is not spontaneously broken. This is obviously wrong in the low temperature phase. The solution to this paradox lies in the fact that the free energy density in the low temperature phase and for an imaginary magnetic field is not defined (it is singular on the imaginary axis of the complex magnetic field plane). It is true that this model is not a vector-like gauge model but in any case verifies all Vafa-Witten conditions, except the existence of the free energy density. This example demonstrates that such an assumption is not trivial and, what is more relevant, to assume the existence of the free energy density is at least at the same level than assume the symmetry be realized in the vacuum.

A possible way to prove Vafa and Witten's claim on parity realization in vector-like theories could be to show the existence of a Transfer Matrix connecting the Euclidean formulation with the Hamiltonian approach in the presence of any hermitian symmetry breaking field. A weaker sufficient condition would be the positivity of $Z(\lambda)$ around $\lambda=0$, even if, from a mathematical point of view, it is not a necessary one for the symmetry to be realized.

The proof of these conditions for any symmetry breaking operator seems very hard, even for the weaker condition. However in the case of the more standard operator $F \tilde{F}$, associated to the $\theta$ vacuum term, the reflection positivity of $\mathcal{Z}$ has been shown for the two dimensional pure gauge model using the lattice regularization scheme [6]. Up to our knowledge a generalization of this result to four dimensional theories and (or) dynamical fermions does not exists. Only arguments suggesting that a consistent Hamiltonian approach could be constructed in the four dimensional pure gauge Yang-Mills model can be found in the literature [7]. Summarizing, even if Vafa and Witten's conjecture seems to be plausible, a theorem on the impossibility to break spontaneously parity in vector-like theories is still lacking.

\section{Acknowledgements}

This work has been partially supported by CICYT (Proyecto AEN97-1680). A.G. was supported by a Istituto Nazionale di Fisica Nucleare fellowship at the University of Zaragoza.

\section{REFERENCES}

1. C. Vafa, E. Witten, Phys. Rev. Lett. 53 (1984) 535.

2. V. Azcoiti, A. Galante, Phys. Rev. Lett. 83 (1999) 1518.

3. K. Binder, Z. Phys. B43, 119 (1981).

4. M. Mezard, G. Parisi and M.A. Virasoro in "Spin Glass Theory and Beyond", World Scientific Lecture Notes in Physics Vol. 9 (1987).

5. V. Azcoiti, V. Laliena, X.Q. Luo, Phys. Lett. B354 (1995) 111.

6. E. Seiler, Gauge Theories as a Problem of Constructive Quantum Field Theory and Statistical Mechanics, Lecture Notes in Physics 159 (Springer-Verlag, Berlin 1982).

7. M. Asorey, P.K. Mitter, On geometry, topology and $\theta$-sectors in the regularized quantum Yang-Mills theory, CERN TH-2423 preprint (1982). 